\title{
Linear Response Theory Around a Localized Impurity in the Pseudogap Regime of an Anisotropic Superconductor
}

\author{
N. Andrenacci, ${ }^{1}$ G. G. N. Angilella, ${ }^{2}$ H. Beck, ${ }^{1}$ and R. Pucci ${ }^{2}$
}

\begin{abstract}
Published online: 27 September 2005
We compare and contrast the polarizability of a $d$-wave superconductor in the pseudogap regime, within the precursor pairing scenario (dPG), and of a $d$-density-wave (dDW) state, characterized by a $d$-wave hidden order parameter, but no pairing. Our study is motivated by STM imaging experiments around an isolated impurity, which may in principle distinguish between precursor pairing and dDW order in the pseudogap regime of the high- $T_{c}$ superconductors. In both cases, the q-dependence of the polarizability is characterized by an azimuthal modulation, consistent with the $d$-wave symmetry of the underlying state. However, only the dDW result shows the fingerprints of nesting, with nesting wave vector $\mathbf{Q}=(\pi, \pi)$, albeit imperfect, due to a nonzero value of the hopping ratio $t^{\prime} / t$ in the band dispersion relation. As a consequence of nesting, the presence of hole pockets is also reflected by the $(\mathbf{q}, \omega)$ dependence of the retarded polarizability.
\end{abstract}

KEY WORDS: high- $T_{c}$ superconductors; impurity effects; STM; pseudogap; hidden order; unconventional density waves.

It has been recently proposed that direct imaging of the local density of states (LDOS) around an isolated impurity by means of scanning tunneling microscopy (STM) [1-4] could help understand the nature of the 'normal' state in the pseudogap regime of the high- $T_{c}$ superconductors (HTS) [5-8]. The idea that an anisotropic superconducting gap should give rise to directly observable spatial features in the tunneling conductance near an impurity was suggested by Byers et al. [9], whereas earlier studies [10] had considered perturbations of the order parameter to occur within a distance of the order of the coherence length $\xi$ around an impurity. Later, it was shown that an isolated impurity in a $d$-wave superconductor produces virtual bound states close to the Fermi level, in the nearly unitary limit [11]. Such a quasi-bound state should appear as a pronounced peak near the

\footnotetext{
${ }^{1}$ Institut de Physique, Université de Neuchâtel, CH-2000 Neuchâtel, Switzerland.

${ }^{2}$ Dipartimento di Fisica e Astronomia Università di Catania, and Lab. MATIS-INFM, and INFN, Sez. Catania, Via S. Sofia, 64, I95123 Catania, Italy.
}

Fermi level in the LDOS at the impurity site [12], as is indeed observed in Bi-2212 [1] and YBCO [13].

In the underdoped regime of the HTS, various models have been proposed to describe the pseudogap state in the temperature range $T_{c}<T<T^{*}$ $[14,15]$. These include the precursor pairing scenario, where the pseudogap is associated to phase fluctuations of the order parameter above $T_{c}$ [16]. Within the precursor pairing scenario, the phase diagram of the HTS can be described as a crossover from BoseEinstein condensation (in the underdoped regime) to BCS superconductivity (in the overdoped regime) [17-20].

Recently, it has been proposed that many properties of the pseudogap regime may be explained within the framework of the so-called $d$-wave-density scenario $(\mathrm{dDW})$ [21-23]. This is based on the idea that the pseudogap regime be characterized by a fully developed order parameter, at variance with the precursor pairing scenario, where a fluctuating order parameter is postulated. The $\mathrm{dDW}$ state is an ordered state of unconventional kind, and is usually associated with staggered orbital currents in the $\mathrm{CuO}_{2}$ square lattice of the HTS [24-27]. Much 
attention has been recently devoted to show the consistency of the dDW scenario with several experimental properties of the HTS [22]. These include transport properties, such as the electrical and thermal conductivities [28,29] and the Hall effect $[30,31]$, thermodynamic properties [32,33], time symmetry breaking [34], and angular resolved photoemission spectroscopy (ARPES) [35]. The possible occurrence of a dDW state in microscopic models of correlated electrons has been checked in ladder networks [36].

In the normal state, the frequency-dependent LDOS at the nearest $(\mathrm{NN})$ and next-nearest neighbor (NNN) sites, with respect to the impurity site, should contain fingerprints of whether the pseudogap regime is characterized by precursor pairing [37] or dDW order [6,7]. This is due to the fact that while pairing above $T_{c}$ without phase coherence is a precursor of cooper pairing, and therefore of spontaneous breaking of $\mathrm{U}(1)$ gauge invariance, the dDW state can be thought as being characterized by the spontaneous breaking of particle-hole symmetry, in the same way as a charge density wave breaks pseudospin SU(2) symmetry [38].

In this context, a complementary information is that provided by the polarizability $F^{\mathrm{R}}(\mathbf{q}, \omega)$ of the system, which gives a measure of the linear response of the charge density to an impurity potential. In the case of $d$-wave superconductors (dSC), it has been demonstrated that the anisotropic dependence of the superconducting order parameter on the wave-vector $\mathbf{q}$ gives rise to a clover-like azimuthal modulation of $F^{\mathrm{R}}(\mathbf{q}, \omega)$ along the Fermi line for a 2D system [39]. These patterns in the $\mathbf{q}$ dependence of $F^{\mathrm{R}}(\mathbf{q}, \omega)$ are here confirmed also for a more realistic band for the cuprates. In addition to that, the dDW result also shows fingerprints of the $\mathbf{Q}=(\pi, \pi)$ nesting properties of such a state.

Within linear response theory, the displaced charge density $\delta \rho(\mathbf{r})$ by a scattering potential $V(\mathbf{r})$ in the Born approximation is given by $[40,41]$

$$
\delta \rho(\mathbf{r})=\int V\left(\mathbf{r}^{\prime}\right) F^{\mathrm{R}}\left(\mathbf{r}-\mathbf{r}^{\prime}, E_{\mathrm{F}}\right) d \mathbf{r}^{\prime},
$$

which implicitly defines the linear response function $F^{\mathrm{R}}\left(\mathbf{r}, E_{\mathrm{F}}\right)$ at the Fermi energy $E_{\mathrm{F}}$. Here and in the following, we set the elementary charge $e=1$. In momentum space, Eq. (1) readily translates into $\delta \rho(\mathbf{q})=$ $V(\mathbf{q}) F^{\mathrm{R}}\left(\mathbf{q}, E_{\mathrm{F}}\right)$, showing that, for a highly localized scattering potential in real space $\left[V(\mathbf{r})=V_{0} \delta(\mathbf{r})\right.$, say], the Fourier transform $\delta \rho(\mathbf{q})$ of the displaced charge is simply proportional to $F^{\mathrm{R}}\left(\mathbf{q}, E_{\mathrm{F}}\right)$.
In the presence of superconducting pairing, the generalization of the linear response function is given by the density-density correlation function (polarizability) [42]:

$$
\begin{aligned}
F\left(\mathbf{q}, i \omega_{v}\right)= & \operatorname{Tr} \frac{1}{\beta} \sum_{\omega_{n}} \frac{1}{N} \sum_{\mathbf{k}} \tau_{3} \mathcal{G}\left(\mathbf{k}, i \omega_{n}\right) \\
& \times \tau_{3} \mathcal{G}\left(\mathbf{k}-\mathbf{q}, i \omega_{n}-i \omega_{v}\right)
\end{aligned}
$$

where $\mathcal{G}\left(\mathbf{k}, i \omega_{n}\right)=\left[i \omega_{n} \tau_{0}+\xi_{\mathbf{k}} \tau_{3}+\Delta_{\mathbf{k}} \tau_{1}\right] /\left[\left(i \omega_{n}\right)^{2}-\right.$ $\left.E_{\mathbf{k}}^{2}\right]$ is the dSC matrix Green's function in Nambu notation, $\beta=T^{-1}$ is the inverse temperature, $\omega_{\nu}=2 v \pi T$ is a bosonic Matsubara frequency, $\tau_{i}$ are the Pauli matrices in spinor space, the summations are performed over the $N$ wave-vectors $\mathbf{k}$ of the first Brillouin zone (1BZ) and all fermionic Matsubara frequencies $\omega_{n}=(2 n+1) \pi T$, and the trace is over the spin indices. (Units are such that $\hbar=k_{\mathrm{B}}=1$ and lattice spacing $a=1$.) Here, $\xi_{\mathbf{k}}=\epsilon_{\mathbf{k}}-\mu$, with $\epsilon_{\mathbf{k}}$ the single-particle dispersion relation:

$$
\epsilon_{\mathbf{k}}=-2 t\left(\cos k_{x}+\cos k_{y}\right)+4 t^{\prime} \cos k_{x} \cos k_{y},
$$

where $t=0.3 \mathrm{eV}, t^{\prime} / t=0.3$ are tight binding hopping parameters appropriate for the cuprate superconductors, and $\mu$ the chemical potential. For a $d$ wave superconductor, we assume a mean-field gap $\Delta_{\mathbf{k}}=\Delta_{\circ} g_{\mathbf{k}}$, with $g_{\mathbf{k}}=\frac{1}{2}\left(\cos k_{x}-\cos k_{y}\right)$ and $E_{\mathbf{k}}=$ $\left(\xi_{\mathbf{k}}^{2}+\Delta_{\mathbf{k}}^{2}\right)^{1 / 2}$. The retarded polarizability is then defined as usual in terms of the analytic continuation as $F^{\mathrm{R}}(\mathbf{q}, \omega)=F\left(\mathbf{q}, i \omega_{\nu} \mapsto \omega+i 0^{+}\right)$. The polarizability in the SC case has been originally studied by Prange [42], later generalized in Ref. [39] for a dSC with isotropic dispersion relation, and in Ref. [43] for a dSC with the more realistic dispersion relation Eq. (3).

In the pseudogap regime, for $T_{c}<T<T^{*}$, within the precursor pairing scenario (dPG) [17], one assumes the existence of cooper pairs characterized by a 'binding energy' $\Delta_{\mathbf{k}}$ having the same symmetry of the true superconducting gap below $T_{c}$, but no phase coherence. In other words, no true offdiagonal long range order develops, and one rather speaks of a 'fluctuating' order [16]. This means that the quasiparticle spectrum $E_{\mathbf{k}}=\left(\xi_{\mathbf{k}}^{2}+\Delta_{\mathbf{k}}^{2}\right)^{1 / 2}$ is still characterized by a pseudogap $\Delta_{\mathbf{k}}=\Delta_{\circ} g_{\mathbf{k}}$ opening at the Fermi energy with $d$-wave symmetry, but now without phase coherence. Therefore, the diagonal elements of the matrix Green's function $\mathcal{G}_{\mathrm{dPG}}\left(\mathbf{k}, i \omega_{n}\right)=$ $\left[i \omega_{n} \tau_{0}+\xi_{\mathbf{k}} \tau_{3}\right] /\left[\left(i \omega_{n}\right)^{2}-E_{\mathbf{k}}^{2}\right]$ coincide with those of its superconducting counterpart, while the off-diagonal, anomalous elements are null. Within this precursor pairing scenario, Eq. (2) in the pseudogap regime 
then evaluates to:

$$
\begin{aligned}
F_{\mathrm{dPG}}\left(\mathbf{q}, i \omega_{\nu}\right)= & \frac{1}{N} \sum_{\mathbf{k}}\left[\left(u_{\mathbf{k}}^{2} u_{\mathbf{k}-\mathbf{q}}^{2}+v_{\mathbf{k}}^{2} v_{\mathbf{k}-\mathbf{q}}^{2}\right)\right. \\
& \times\left(\frac{f\left(E_{\mathbf{k}}\right)-f\left(E_{\mathbf{k}-\mathbf{q}}\right)}{E_{\mathbf{k}}-E_{\mathbf{k}-\mathbf{q}}-i \omega_{\nu}}+\text { H.c. }\right) \\
& +\left(u_{\mathbf{k}-\mathbf{q}}^{2} v_{\mathbf{k}}^{2}+u_{\mathbf{k}}^{2} v_{\mathbf{k}-\mathbf{q}}^{2}\right) \\
& \left.\times\left(\frac{f\left(E_{\mathbf{k}}\right)+f\left(E_{\mathbf{k}-\mathbf{q}}\right)-1}{E_{\mathbf{k}}+E_{\mathbf{k}-\mathbf{q}}-i \omega_{\nu}}+\text { H.c. }\right)\right] .
\end{aligned}
$$

As anticipated earlier, the dDW state [21] is characterized by a broken symmetry and a welldeveloped order parameter $\left\langle c_{\mathbf{k}+\mathbf{Q} s}^{\dagger} c_{\mathbf{k} s^{\prime}}\right\rangle=i \Phi_{\mathbf{Q}} g_{\mathbf{k}} \delta_{s s^{\prime}}$, at variance with the precursor pairing scenario of the pseudogap regime. $[\mathbf{Q}=(\pi, \pi)$ is the $\mathrm{dDW}$ ordering wave-vector.] Such a state is associated to staggered orbital currents circulating with alternating sense in the neighboring plaquettes of the underlying square lattice. As a result, the unit cell in real space is doubled, and the Brillouin zone is correspondingly halved. The mean-field Hamiltonian for the dDW state can be conveniently rewritten as [28,29]:

$$
H_{\mathrm{dDW}}=\sum_{\mathbf{k} s}^{\prime} \Psi_{\mathbf{k} s}^{\dagger}\left[\left(\epsilon_{\mathbf{k}}^{+}-\mu\right) \tau_{0}+\epsilon_{\mathbf{k}}^{-} \tau_{3}+D_{\mathbf{k}} \tau_{1}\right] \Psi_{\mathbf{k} s},
$$

where $\Psi_{\mathbf{k} s}^{\dagger}=\left(c_{\mathbf{k} s}^{\dagger} c_{\mathbf{k}+\mathbf{Q} s}^{\dagger}\right), \quad \epsilon_{\mathbf{k}}^{ \pm}=\frac{1}{2}\left(\epsilon_{\mathbf{k}} \pm \epsilon_{\mathbf{k}+\mathbf{Q}}\right), \quad D_{\mathbf{k}}=$ $D_{\circ} g_{\mathbf{k}}$ is the dDW 'pseudogap', and the prime restricts the summation over wave-vectors $\mathbf{k}$ belonging to the reduced ('magnetic') Brillouin zone only. Correspondingly, the matrix Green's function at the imaginary time $\tau$ can be defined as $\mathcal{G}_{\mathrm{dDW}}(\mathbf{k}, \tau)=$ $-\left\langle T_{\tau} \Psi_{\mathbf{k} s}(\tau) \Psi_{\mathbf{k s}}^{\dagger}(0)\right\rangle$, whose inverse reads [7,29]:

$$
\mathcal{G}_{\mathrm{dDW}}^{-1}\left(\mathbf{k}, i \omega_{n}\right)=\left(\begin{array}{ll}
i \omega_{n}-\xi_{\mathbf{k}} & i D_{\mathbf{k}} \\
-i D_{\mathbf{k}} & i \omega_{n}-\xi_{\mathbf{k}+\mathbf{Q}}
\end{array}\right) .
$$

The case of perfect nesting $\left(t^{\prime}=0\right)$ has been explicitly studied in Ref. [29]. In the general case $\left(t^{\prime} \neq 0\right)$, one finds

$$
\begin{aligned}
\mathcal{G}_{\mathrm{dDW}}\left(\mathbf{k}, i \omega_{n}\right)= & \frac{1}{\left(i \omega_{n}-E_{\mathbf{k}}^{+}\right)\left(i \omega_{n}-E_{\mathbf{k}}^{-}\right)} \\
& \times\left(\begin{array}{ll}
i \omega_{n}-\xi_{\mathbf{k}+\mathbf{Q}} & -i D_{\mathbf{k}} \\
i D_{\mathbf{k}} & i \omega_{n}-\xi_{\mathbf{k}}
\end{array}\right),
\end{aligned}
$$

where $E_{\mathbf{k}}^{ \pm}=-\mu+\epsilon_{\mathbf{k}}^{+} \pm \sqrt{\left(\epsilon_{\mathbf{k}}^{-}\right)^{2}+D_{\mathbf{k}}^{2}}$ are the two branches of the quasiparticle spectrum obtained by diagonalizing Eq. (5) [28]. Notice that $E_{\mathbf{k}+\mathbf{Q}}^{ \pm}=E_{\mathbf{k}}^{ \pm}$.
The polarizability in the dDW state has been derived in Ref. [43], and can be cast in compact matrix notation as:

$$
\begin{aligned}
F_{\mathrm{dDW}}\left(\mathbf{q}, i \omega_{\nu}\right)= & \operatorname{Tr} \frac{1}{\beta} \sum_{\omega_{n}} \frac{1}{N} \sum_{\mathbf{k}}^{\prime} \kappa \mathcal{G}_{\mathrm{dDW}}\left(\mathbf{k}, i \omega_{n}\right) \\
& \times \kappa \mathcal{G}_{\mathrm{dDW}}\left(\mathbf{k}-\mathbf{q}, i \omega_{n}-i \omega_{\nu}\right),
\end{aligned}
$$

where now $\kappa=\tau_{0}+\tau_{1}$, and $\mathcal{G}_{\mathrm{dDW}}$ is the matrix Green's function for the dDW state, Eq. (7). Performing the frequency summation, one eventually finds:

$$
F_{\mathrm{dDW}}\left(\mathbf{q}, i \omega_{\nu}\right)=\frac{1}{N} \sum_{\mathbf{k}}^{\prime} \sum_{i, j= \pm} \frac{f\left(E_{\mathbf{k}}^{i}\right)-f\left(E_{\mathbf{k}-\mathbf{q}}^{j}\right)}{E_{\mathbf{k}}^{i}-E_{\mathbf{k}-\mathbf{q}}^{j}-i \omega_{\nu}} .
$$

The case of competition between dSC and dDW orders has been also considered in Ref. [43], where the polarizability is shown to have an expression similar to Eq. 9 (see also Ref. [44] for a discussion on dSC and dDW).

We have evaluated numerically the polarizability for the dPG and the dDW cases, Eqs. (4) and (9), as a function of the relevant variables. Our numerical results for the wave-vector dependence of the static polarizability $F(\mathbf{q}, 0)$ over the $1 \mathrm{BZ}$ in the dPG and in the pure dDW cases are shown in Fig. 1. As a result of the $d$-wave symmetry of both the pseudogap within the precursor pairing scenario, and of the dDW order parameter, $F(\mathbf{q}, 0)$ is characterized by a four-lobed pattern or azimuthal modulation [39]. However, the dDW case is also characterized by the presence of 'hole pockets,' centered around $\mathbf{Q} / 2=(\pi / 2, \pi / 2)$ and symmetry-related points, due to the (albeit imperfect) nesting properties of the dDW state, with nesting vector $\mathbf{Q}=(\pi, \pi)$ (Fig. 1, right). Such a feature is reflected in the $\mathbf{q}$ dependence of $F_{\mathrm{dDw}}(\mathbf{q}, 0)$, which is characterized by local maxima at the hole pockets.

As a result, the Fourier transform in real space of the polarizability, $F(\mathbf{r}, 0)$, is characterized by Friedel-like oscillations as $|\mathbf{r}|$ increases from the impurity site, as expected [39,45]. These radial, damped oscillations are then superimposed by an azimuthal modulation, due to the $d$-wave symmetry of the normal state, both in the dPG and in the dDW cases [39]. One finds a checkerboard pattern, closely reflecting the symmetry of the underlying lattice, with local maxima on the $\mathrm{NN}$ and local minima on the NNN sites. Since these features are common to both the $\mathrm{dPG}$ and dDW cases, the spatial dependence of the charge density oscillations is not directly helpful in distinguishing between the dPG and dDW states. 

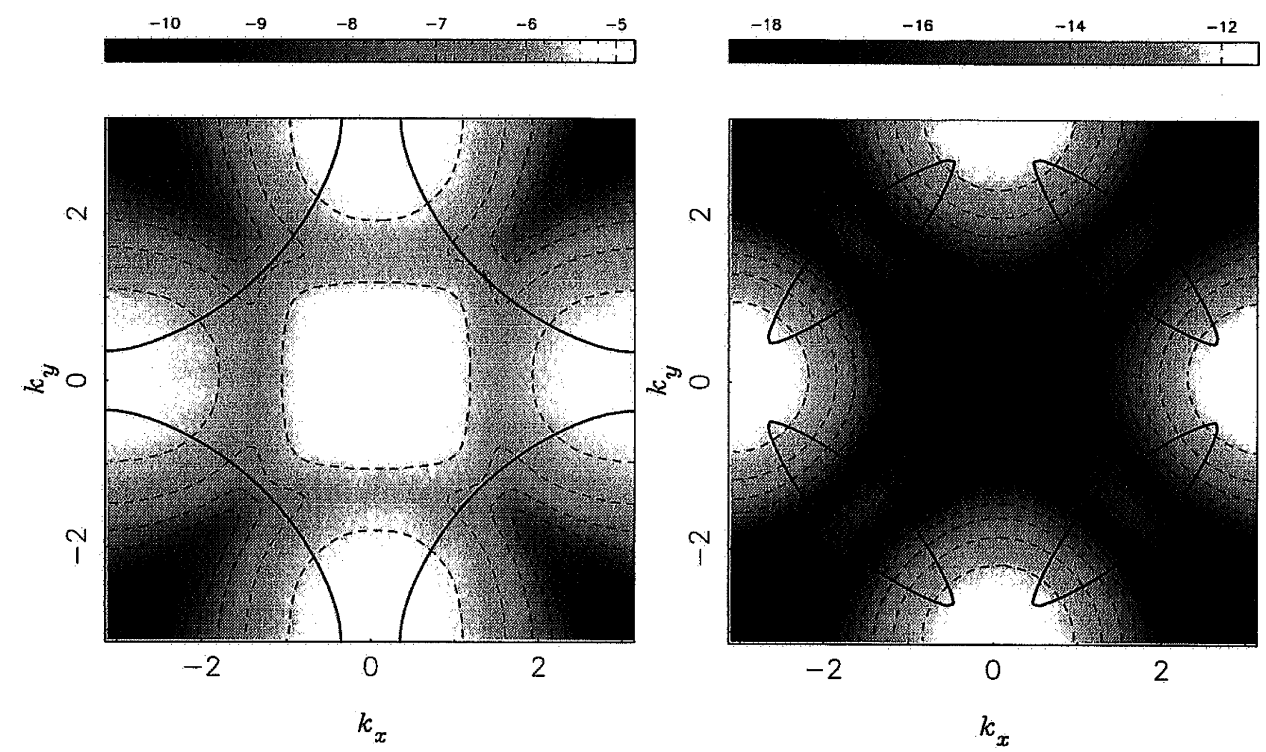

Fig. 1. Static polarizability $F(\mathbf{q}, 0)\left[\mathrm{eV}^{-1}\right]$ in the $1 \mathrm{BZ}$ for the $\mathrm{dPG}($ left $)$ and $\mathrm{dDW}$ states $(r i g h t)$. Numerical parameters are $t=0.3 \mathrm{eV}, t^{\prime} / t=0.3, \mu=-t$, corresponding to a hole-like Fermi line and a hole doping $\sim 14.3 \%, \Delta_{\circ}=D_{\circ}=0.06 \mathrm{eV}$ in the $\mathrm{dPG}$ and in the dDW cases, respectively [35], and $T=100 \mathrm{~K}$. Thick solid lines are the Fermi line $\xi_{\mathbf{k}}=0(\mathrm{dPG}$, left $)$, and the locus $E_{\mathbf{k}}^{-}=0(\mathrm{dDW}$, right), the latter enclosing four 'hole pockets' around the point $\mathbf{Q} / 2$, and symmetry-related points.

However, the $\mathbf{r}$ and $\mathbf{q}$ dependences of several quantities of interest for STM studies can be easily connected by means of Fourier transform scanning tunneling microscopy (FT-STM) techniques (see, e.g., Ref. [46], and references therein, and Ref. [47] for the relevance of FT-STM in detecting fluctuating stripes in the HTS).

In summary, motivated by recent STM experiments around a localized impurity in the HTS, we have derived the polarizability $F(\mathbf{q}, \omega)$ for the pseudogap phase, both in the precursor pairing scenario and in the $\mathrm{dDW}$ scenario.

In the static limit, the $\mathbf{q}$ dependence of $F(\mathbf{q}, 0)$ reflects the $d$-wave symmetry of the precursor pairing 'pseudogap' or of the dDW order parameter, with an azimuthal modulation consistent with a cloverlike pattern, as expected also for a superconductor with an isotropic band [39]. However, at variance to the dPG case, the $q$ dependence of the static polarizability in the dDW state clearly exhibits the presence of hole pockets, due to the (albeit imperfect) nesting properties of the $\mathrm{dDW}$ state, with nesting vector $\mathbf{Q}=$ $(\pi, \pi)$. Qualitatively similar results to the pure dDW case are obtained also in the mixed $\mathrm{dSC}+\mathrm{dDW}$, thus showing that hole pockets are a distinctive feature of dDW order [43]. Such a behavior is confirmed by the $\mathbf{r}$ dependence of the static polarizability in real space. An analysis of the frequency dependence of the retarded polarizability $F^{\mathrm{R}}(\mathbf{q}, \omega)$ reveals that the $\mathbf{q}$ evolution of the features (local maxima or shoulders) in the $\omega$ dependence of this function is closely connected with the relative position of wave-vector $\mathbf{q}$ with respect to the Fermi line, and is therefore sensitive to the possible presence of hole pockets, as is the case for the dDW state [43].

\section{ACKNOWLEDGMENTS}

We are indebted with L. Benfatto, P. Castorina, J. O. Fjærestad, F. E. Leys, V. M. Loktev, N. H. March, M. Salluzzo, S. G. Sharapov, F. Siringo, D. Zappalá for stimulating discussions and correspondence.

\section{REFERENCES}

1. S. H. Pan, E. W. Hudson, K. M. Lang, H. Eisaki, S. Uchida, and J. C. Davis, Nature (London) 403, 746 (2000).

2. E. W. Hudson, S. H. Pan, A. K. Gupta, K. W. Ng, and J. C. Davis, Science 285, 88 (1999).

3. E. W. Hudson, K. M. Lang, V. Madhavan, S. H. P. H. Eisaki, S. Uchida, and J. C. Davis, Nature (London) 411, 920 (2001). 
4. S. H. Pan, J. P. O'Neal, R. L. Badzey, G. Chamon, H. Ding, J. R. Engelbrecht, Z. Wang, H. Eisaki, S. Uchida, A. K. Guptak, K. W. Ng, E. W. Hudson, K. M. Lang, and J. C. Davis, Nature (London) 413, 282 (2001).

5. J. Zhu, W. Kim, C. S. Ting, and J. P. Carbotte, Phys. Rev. Lett. 87, 197001 (2001).

6. Q. Wang, Phys. Rev. Lett. 88, 057002 (2002).

7. D. K. Morr, Phys. Rev. Lett. 89, 106401 (2002).

8. B. Møller Andersen, Phys. Rev. B 68, 094518 (2003).

9. J. M. Byers, M. E. Flatte, and D. J. Scalapino, Phys. Rev. Lett. 71, 3363 (1993).

10. E. S. Choi and P. Muzikar, Phys. Rev. B 41, 1812 (1990).

11. A. V. Balatsky, Phys. Rev. B 51, 15547 (1995).

12. M. I. Salkola, A. V. Balatsky, and D. J. Scalapino, Phys. Rev. Lett. 77, 1841 (1996).

13. M. Iavarone, M. Salluzzo, R. Di Capua, M. G. Maglione, R. Vaglio, G. Karapetrov, W. K. Kwok, and G. Crabtree, Phys. Rev. B 65, 214506 (2002).

14. M. Randeria, in Proceedings of the CXXXVI International School of Physics "E. Fermi" on "Models and Phenomenology for Conventional and High-Temperature Superconductivity", G. Iadonisi, R. J. Schrieffer, and M. L. Chiofalo, eds. (IOS, Amsterdam, 1999), preprint cond-mat/9710223.

15. T. Timusk and B. Statt, Rep. Prog. Phys. 62, 61 (1999).

16. V. J. Emery and S. A. Kivelson, Nature (London) 374, 434 (1995).

17. V. M. Loktev, R. M. Quick, and S. G. Sharapov, Phys. Rep. 349, 1 (2001)

18. M. Randeria, in Base Einstein Condensation, A. Griffin, D. Snoke, and S. Stringari, eds. (Cambridge University Press, Cambridge, 1995), p. 355.

19. N. Andrenacci, A. Perali, P. Fieri, and G. C. Strinati, Phys. Rev. B 60, 12410 (1999).

20. G. C. Strinati, Phys. Essays 13, 427 (2000).

21. S. Chakravarty, R. B. Laughlin, D. K. Morr, and C. Nayak, Phys. Rev. B 63, 094503 (2001).

22. S. Chakravarty, H. Kee, and C. Nayak, Int. J. Mod. Phys. B 15, 2901 (2001).

23. S. Chakravarty and H.-Y. Kee, Phys. Rev. B 61, 14821 (2000).

24. I. Affleck and J. B. Marston, Phys. Rev. B 37, 3774 (1988).

25. J. B. Marston and I. Affleck, Phys. Rev. B 39, 11538 (1989).
26. G. Kotliar, Phys. Rev. B 37, 3664 (1988).

27. H. Schulz, Phys. Rev. B 39, 2940 (1989).

28. X. Yang and C. Nayak, Phys. Rev. B 65, 064523 (2002).

29. S. G. Sharapov, V. P. Gusynin, and H. Beck, Phys. Rev. B 67, 144509 (2003).

30. S. Chakravarty, C. Nayak, S. Tewari, and X. Yang, Phys. Rev. Lett. 89, 277003 (2002).

31. F. F. Balakirev, J. B. Betts, A. Migliori, S. Ono, Y. Ando, and G. S. Boebinger, Nature (London) 424, 912 (2003).

32. H.-Y. Kee and Y. B. Kim, Phys. Rev. B 66, 012505 (2002).

33. C. Wu and W. V. Liu, Phys. Rev. B 66, R020511 (2002).

34. A. Kaminski, S. Rosenkranz, H. M. Fretwell, J. C. Campuzano, Z. Li, H. Raffy, W. G. Cullen, H. You, C. G. Olson, C. M. Varma, and H. Höchst, Nature (London) 416, 610 (2002).

35. S. Chakravarty, C. Nayak, and S. Tewari, Phys. Rev. B 68 , R100504 (2003).

36. J. B. Marston, J. O. Fjærestad, and A. Sudb $\varnothing$, Phys. Rev. Lett. 89, 056404 (2002).

37. H. V. Kruis, I. Martin, and A. V. Balatsky, Phys. Rev. B 64, $054501(2001)$

38. S. Shen and X. C. Xie, Physica B 230-232, 1061 (1997), also available as preprint cond-mat/9507075.

39. G. G. N. Angilella, F. E. Leys, N. H. March, and R. Pucci, J. Phys. Chem. Solids 64, 413, 1431 (2003).

40. J. C. Stoddart, N. H. March, and M. J. Stott, Phys. Rev. 186, 683 (1969).

41. W. Jones and N. H. March, Theoretical Solid-State Physics. Non-equilibrium and Disorder, vol. 2 (Dover, New York, 1986).

42. R. E. Prange, Phys. Rev. 129, 2495 (1963).

43. N. Andrenacci, G. G. N. Angilella, H. Beck, and R. Pucci, Phys. Rev. B 70, 024507 (2004).

44. L. Benfatto, S. G. Sharapov, and H. Beck, Eur. Phys. J. B 39, 469 (2004).

45. J. Zhu and C. S. Ting, Phys. Rev. Lett. 87, 147002 (2001).

46. L. Capriotti, D. J. Scalapino, and R. D. Sedgewick, Phys. Rev. $B$ 68, 014508 (2003)

47. S. A. Kivelson, I. P. Bindloss, E. Pradkin, V. Oganesyan, J. M. Tranquada, A. Kapitulnik, and C. Howald, Rev. Mod. Phys. 75, 1201 (2003). 